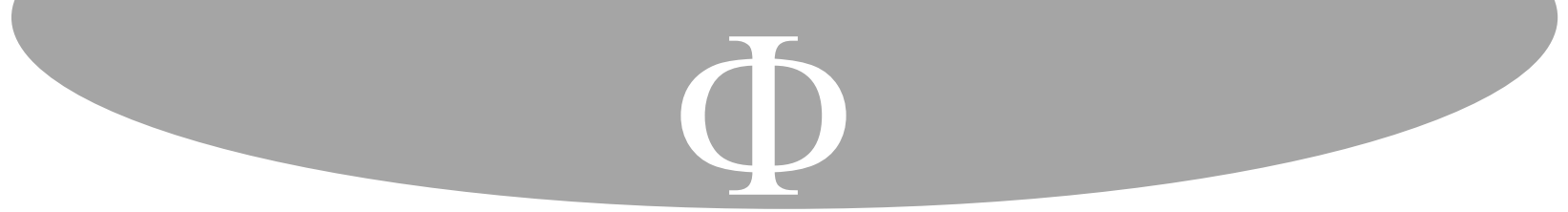

\title{
Identidad de la universidad franciscana en Colombia. Un estudio en perspectiva pedagógica*
}

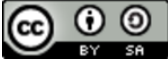

Para citar este artículo: Vanegas Carvajal, Edgar Alonso. «Identidad de la universidad franciscana en Colombia. Un estudio en perspectiva pedagógica». Franciscanum 176, Vol. 63 (2021): 1-28.

\section{Resumen}

El objetivo de este artículo es mostrar el compromiso de la pedagogía franciscana en la construcción de identidad de la Universidad Franciscana en Colombia. Es decir, se busca indagar acerca de la responsabilidad que tiene la pedagogía franciscana con la identidad en cuatro universidades franciscanas de Colombia. Para ello, se proponen tres escenarios interconectados: se parte de la relación entre franciscanismo y universidad que emerge desde la primera fraternidad franciscana; seguidamente, se analiza la concepción de pedagogía franciscana que declara cada una de las universidades franciscanas de Colombia; finalmente, se proponen líneas y acciones de cooperación interinstitucional entre las universidades franciscanas de Colombia, encaminadas a responder a los retos y desafíos que la globalización le plantea a la universidad moderna.

La investigación es eminentemente documental-descriptiva con enfoque cualitativo de tipo no experimental, puesto que prevaleció un análisis crítico a partir de la revisión y análisis de material bibliográfica seleccionado intencionalmente. En consecuencia, se utilizó como técnica el análisis categorial en los términos propuestos por Corbin y Strauss ${ }^{1}$, que parte de un análisis categorial seguido de un estudio comparativo con muestreo teórico.

\footnotetext{
* Artículo derivado del proyecto de investigación «La pedagogía franciscana como aporte a la construcción de identidad en la Universidad Franciscana en Colombia», aprobado por convocatoria interna de la Universidad de San Buenaventura, seccional Medellín, mediante acta de registro M8830, para el período enero-diciembre de 2020. Un avance del artículo ha sido presentado como ponencia en el «I Congreso Internacional de pensamiento ético. Una mirada interdisciplinaria», en la Universidad CESMAG, de San Juan de Pasto, realizado del 16 al 19 de noviembre de 2020.

** Ph.D. en Filosofía, Universidad Pontificia Bolivariana, Especialista en Pedagogía y Docencia Universitaria, Licenciado en filosofía y teología, Universidad de San Buenaventura Bogotá. Docente investigador de la Universidad de San Buenaventura, Medellín, docente de la Universidad Pontificia Bolivariana, Medellín. Integrante de los grupos de investigación GIDPAD y GIEB. ORCID: https://orcid.org/0000-0001-6124-3944 Contacto: edgar.vanegas@ usbmed.edu.co; vanegasc74@gmail.com.

${ }^{1}$ Juliet Corbin and Anselmo Strauss, Basic of qualitative research: Techniques and procedures for developing grounded theory (Newbury Park, California: SAGE Publications, Inc., 2014), 17.
} 


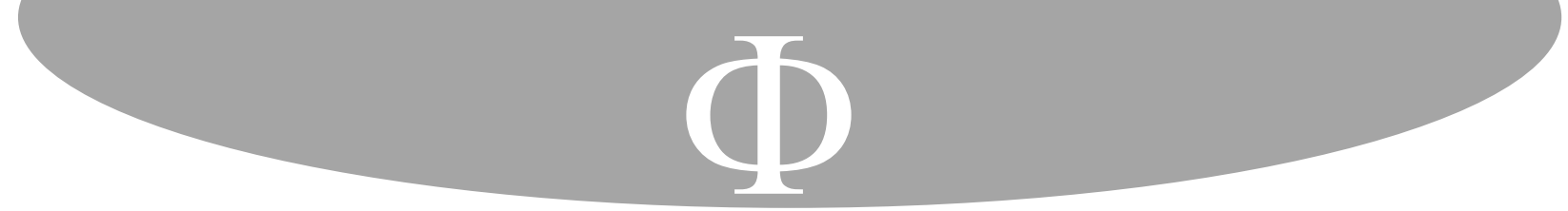

privilegiado para preparar a los hermanos adecuadamente para la evangelización, pero también medio óptimo para realizar la misma obra de la evangelización ${ }^{8}$.

Ahora bien, la relación entre franciscanismo y universidad no ha llegado libre de disputas y querellas, no solo en los siglos XIII y XIV, sino que se han extendido hasta la actualidad, a causa de las tensiones presentes en la Orden Franciscana desde sus inicios, entre quienes privilegian una vuelta a la más genuina observancia evangélica vivida por Francisco y la primitiva comunidad franciscana, y quienes buscan resignificar la experiencia $i l$ Poverello y su primitiva comunidad a los retos y desafíos que nos plantea el mundo contemporáneo.

La historia del franciscanismo nos muestra con hechos concretos y con nombres propios las visiones tan diversas que se vivían al interior del movimiento. Esto ha sido caracterizado por los historiadores como conflictus, sabiendo nosotros hoy que la palabra denota el derecho al disenso, a la opinión distinta, a la aceptación madura de la diferencia y lo diferente; encontrando que, en la Edad Media, según un léxico temático de filosofía medieval, el vocablo transciende el sentido bélico para significar la confrontación de pensamientos, esto es la disputatio, como una herramienta dialógica que da un matiz muy importante al debate académico; que en lugar de ser señal de división, constituyen una línea de continuidad-discontinuidad que se mueven entre la vivencia radical del evangelio a la manera de Francisco de Asís y la actualización de ese modus vivendi al mundo moderno.

Dicho de otra manera: las tensiones presentadas al interior del movimiento franciscano, más allá de presentarse como rupturas contradictorias discontinuas, son expresión de una diversidad poliédrica ${ }^{9}$, signo de un espíritu abierto, pluridimensional y multirreferencial, pero cuya base genética ha de ser en todo caso, Francisco de Asís y el más genuino franciscanismo. En efecto, las instituciones de educación superior de la Orden Franciscana en todo el mundo, convencidas de su tarea de ser transmisora de la cultura, de principios y de valores humanos, cristianos y franciscanos, no han cesado en su empeño de cumplir con la misión de difundir la orto-praxis franciscana a través de las funciones sustantivas de docencia, investigación y proyección a la comunidad.

Dentro de este horizonte de pensamiento franciscano, se encuentran énfasis y campos de acción muy concretos de cada institución que aportan de manera significativa a la resolución de problemas reales de contexto; verbigratia en áreas como la ecología y el medio ambiente; la educación y la pedagogía; la filosofía del lenguaje y la estética; la teología dogmática y las humanidades; la paz y la resolución de conflictos; por mencionar solo algunos de ellos. En este sentido, la presencia del pensamiento franciscano en la universidad no solamente le da continuidad a una línea de pensamiento que a lo largo de la historia ha mantenido un singular modo de interpretar y dar solución de manera auténtica y novedosa a los problemas reales de contexto, sino que, cristaliza y transmite a las nuevas generaciones los valores humanos y franciscanos aportando de manera significativa a la transmisión de la

\footnotetext{
${ }^{8}$ Adolfo Galeano Atehortúa, La Universidad Franciscana. Evangelización y Posmodernidad, 13.

${ }^{9}$ Esta palabra viene el griego poluedron de la raíz poluz (muchos) y edra (base, asiento, cara).
} 

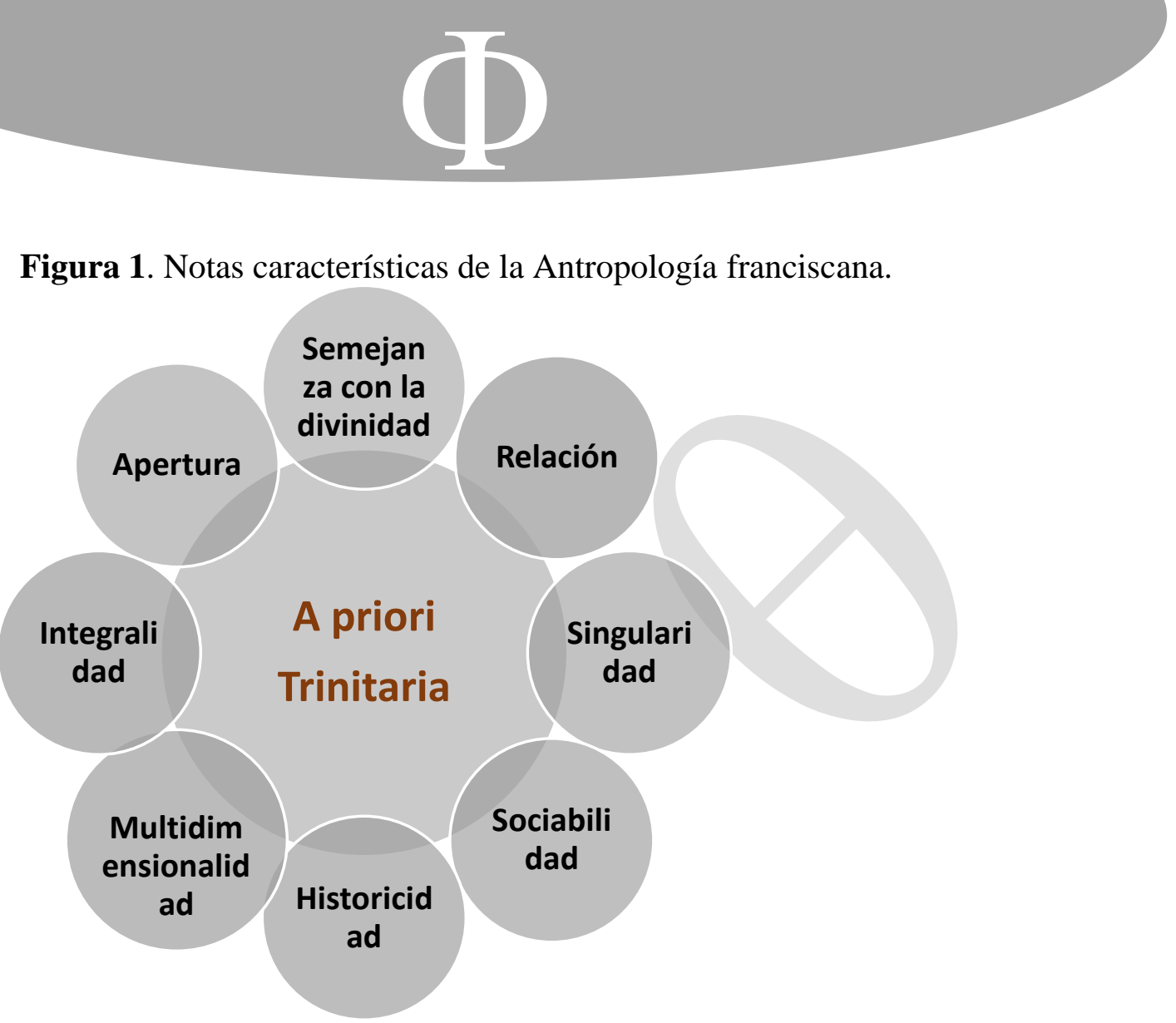

Fuente. Elaboración propia.

\section{Compromiso de la pedagogía Franciscana con la identidad de las Universidades Franciscanas en Colombia}

\subsection{Algo sobre pedagogía franciscana}

La pedagogía franciscana viene constituyendo un tema central de debate en el campo de la educación franciscana, pero no por ello libre de discusiones y cuestionamientos ${ }^{21}$. En efecto, es un tema que despierta distintas tendencias y opiniones, que van desde las más radicales, que niegan que se pueda hablar con propiedad de una pedagogía propiamente franciscana, pasando por una postura restringida, que acepta algún tipo de pedagogía Franciscana, hasta quienes declaran explícitamente que es posible hablar de manera explícita de una pedagogía Franciscana, con una propuesta formativa, unos principios pedagógicos propios y auténticos, que se fundamenta en la primitiva fraternidad franciscana y toman forma con las tesis que nos proponen los maestros de la primera generación franciscana.

\footnotetext{
${ }^{21}$ Es como si los largos y amplios debates alrededor de la pedagogía y su estatuto epistemológico, hayan permeado los debates alrededor de la pedagogía franciscana. Para intentar mitigar de momento este debate, aquí comprendemos la educación, en tanto objeto de estudio de la pedagogía, como un fenómeno complejo que tiene lugar en todos los ámbitos sociales y que no sólo debe ser abordado por las diferentes disciplinas, sino que, principalmente, requiere un abordaje multirreferencial, multidimensional e inter/transdisciplinar, que se caracteriza por establecer diversos tipos y niveles de relación a partir de los principios de apertura, pertinencia, responsabilidad y eticidad. De esta manera hablamos de ciencias de la educación como un conjunto de disciplinas que estudian, describen, analizan y explican los fenómenos socioculturales y educativos desde múltiples perspectivas.
} 


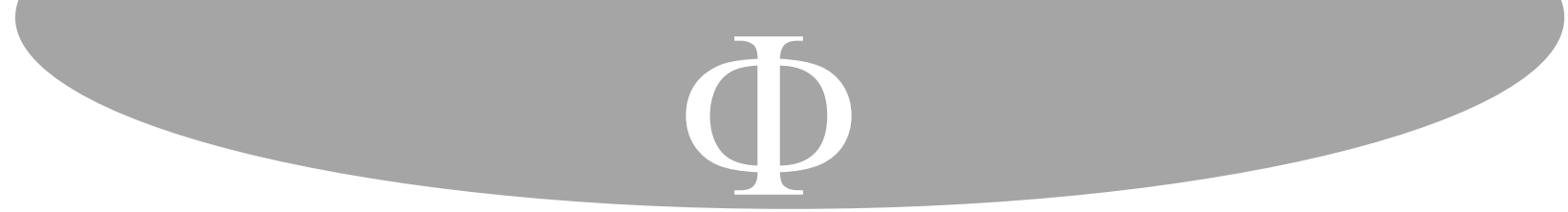

En esta comunicación se parte del hecho que «el discurso franciscano desde su dimensión educativa y formativa ocupa un lugar dentro del campo conceptual de la pedagogía ${ }^{22}$, por lo menos por tres razones:

1. El acervo filosófico, teológico y humanista franciscano, configura un marco teórico y conceptual adecuado para fundamentar el discurso y práctica educativo en clave franciscana.

2. La evangelización que los maestros y maestras franciscanos han ejercido en el ámbito educativo por más de ocho siglos, (en Europa desde 1224; y en América desde $1523^{23}$ ), ha permitido configurar un tipo de pedagogía con identidad franciscana.

3. La larga y amplia trayectoria que los franciscanos han tenido en los centros educativos en todos los niveles y en los diversos contextos, legitiman las prácticas y discursos pedagógicos con identidad propia.

Estas justificaciones se sustentan en un marco referencial suficiente; parece conveniente, aunque sea brevemente, esbozar la idea de pedagogía franciscana que algunos autores han desarrollado, y que han influenciado en nuestro contexto colombiano.

Primeramente, hay que traer al estrado al franciscano y pedagogo Madariaga, cuando precisa que la pedagogía en clave francisana, tiene un significado mucho más amplio e incluyente que el que tradicionalmente suele dársele; puesto que no se debe limitar a la enseñanza institucionalizada, sino que se debe aplicar también a los cuarteles, los reformatorios, las cárceles, los talleres industriales, los orfanatos, los seminarios y noviciados religiosos, «puesto que todos estos centros de educación tienen sus problemas específicos, que requieren un tratamiento adecuado» ${ }^{24}$. Esta idea ha sido actualizada en nuestros días por el pedagogo colombiano Armando Zambrano, cuando afirma: «desde la perspectiva epistemológica, las ciencias de la educación no se limitan al campo escolar ni a la sola formación del profesorado, sus temas de investigación son tan plurales como los enfoques disciplinarios que habitan amistosamente en su seno» ${ }^{25}$.

Esta concepción de pedagogía conlleva una idea de educación abierta, incluyente, flexible y dinámica que, no solo visibiliza, sino que empodera a todos los sectores de la social

\footnotetext{
${ }^{22}$ Carlos Mario Cardona et al, eds., La Paideia Francisana: una mirada a la expansión humana (Medellín: Departamento de Publicaciones USB, 2007), 16.

${ }^{23}$ En América se reconoce que «la Orden Franciscana es la fundadora de la pedagogía en el Nuevo Mundo» por medio del hermano Pedro de Gante, fundador e impulsador de la primera Universidad en América, colegio de Santa Cruz en Tilatelalco, México, en el año de 1523, considerada la primera Universidad americana. Por eso es llamado el primer gran educador de América. Cf. Adolfo Galeano Atehortúa, La Universidad Franciscana. Evangelización y posmodernidad, 14.

${ }^{24}$ Bernardo Madariaga, «La pedagogía franciscana y el actual momento pedagógico», Verdad y Vida: Revista de las ciencias del Espíritu Vol. 4 (1946): 180.

${ }^{25}$ Armando Zambrano Leal, De la pedagogía a las ciencias de la educación: Debates y tránsitos (Barcelona: Universitat Oberta de Catalunya, 2019), 112.
} 


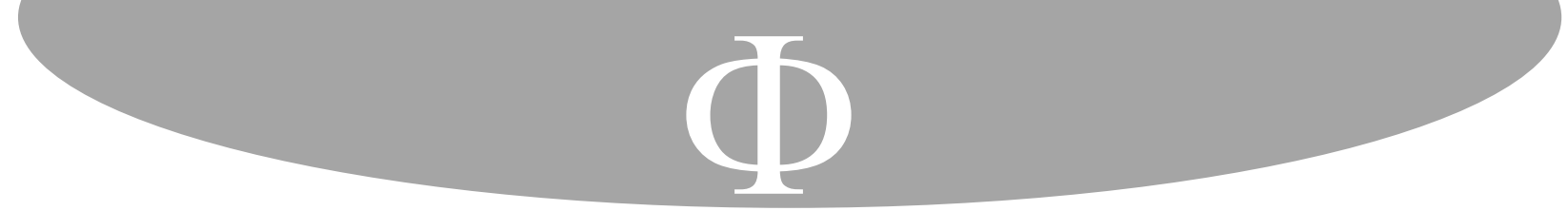

primeros discípulos, reconociendo, al mismo tiempo, los valores personales de cada uno ${ }^{29}$.

Finalmente, el restaurador de la Universidad de San Buenaventura en Colombia, Darío Correa, llega a decir que «la pedagogía franciscana no es ni un método de enseñanza, ni un sistema de educación, sino la vivencia de un Espíritu, el Espíritu de San Francisco de Asís"30; y Benjamín Soto, destacado franciscanista, completa esta visión agregando que, "Francisco sin ser un ilustrado académico ni un pedagogo de escuela, con su vida y comportamiento origina toda una manera de actuar, de ver y de pensar, hoy diríamos: una escuela pedagógica a favor del otro» ${ }^{31}$. Quizás podríamos decir que Francisco fue un pedagogo si concebimos la pedagogía como arte, esto es como creación, como camino, como proyecto, como acompañamiento. Dicho de otra manera: como un modo práctico de conducir al estudiante-persona, que implica una relación de caminar juntos mediados por múltiples (des)encuentros, todos de distinto nivel.

Muchos otros referentes teóricos y experiencias podrían ayudarnos a dilucidar la idea de pedagogía franciscana, basta con mencionar algunos trabajos investigativos a modo de guisa de este apartado: La pedagogía de la evangelización franciscana en el virreinato de Nueva España (siglo XVI): el testimonio de Fray Diego Valadés (2013), por Delfín OrtegaSánchez; Fundamentos filosóficos de una pedagogía franciscana (2007), por Daniel Herrera Restrepo; El lugar del discurso franciscano dentro del campo conceptual de la pedagogía (2007), por Jair Álvarez Torres; La pedagogía francisana como modelo educativo (2012), por Luis Ernesto Chaves Martínez (Tesis doctoral); Homo Viator, L'uomo alla luce della storia della salvezza, Un'antropologia teológica in prospettiva francescana (2008), por Johannes. B Freyer, entre otros.

\subsubsection{Postulados de la pedagogía franciscana}

Para una paideia auténticamente franciscana, todas las personas, las culturas y las religiones, son interlocutoras válidas (legítimas), aunque su visión del hombre, del mundo y de Dios sean diametralmente opuesta a la nuestra; lo que cuenta es que seamos capaces de encontrarnos y sentarnos a dialogar con ellas; una pedagogía franciscana depende en gran medida de la participación que le ofrezcamos al otro en nuestros proyectos.

La pedagogía moderna atribuye al maestro la tarea de acompañar al estudiante, animarlo y sostenerlo en sus esfuerzos por su formación mediante un diálogo continuo. Fue precisamente esto lo que realizó Francisco de Asís, reconociendo los valores de cada hermano y encaminándolo a su plena realización personal dentro de la fraternidad. Los PEI de las cuatro universidades franciscanas de Colombia, refieren en términos más o menos similares, algunas notas pedagógicas que caracterizan los discursos y prácticas en dichas instituciones.

\footnotetext{
${ }^{29}$ Alberto Montealegre González, «Disertación sobre pedagogía franciscana», 15.

${ }^{30}$ Darío Correa Gómez, «Algo sobre pedagogía Franciscana», Itinerario Educativo Vol. 43-44 (2008): 45.

${ }^{31}$ Benjamín Soto Forero, «Hacia una pedagogía franciscana», Revista El Ágora USB Vol. 8 (2008): 462.
} 


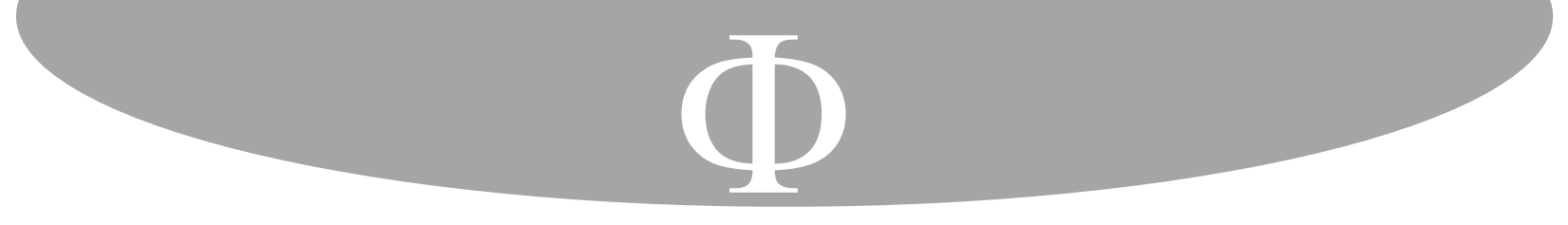

de aprendizaje y con énfasis en lo integral. Unicesmag, habla de modo explícito de una pedagogía franciscana y capuchina que contiene los elementos necesarios para formar «hombres nuevos para tiempos nuevos» ${ }^{46}$ a partir de procesos de personalización y humanización en los términos propuestos por el padre Luis de Castellana.

Por último, Unimar ha entendido que para responder a los nuevos retos y desafíos que le plantea el mundo actual a la Universidad, se requiere transformar la cultura pedagógica de sus procesos formativos en diversos espacios, con el fin de situar «la tarea del estudiante como sujeto responsable de su aprendizaje y como protagonista central de la formación (...), así como la necesidad de situar el texto pedagógico en el contexto específico de nuestra institución y de nuestra experiencia docente» ${ }^{47}$. De esta manera, y vinculando discursos, reflexiones y prácticas docentes Unimar entiende la pedagogía,

como un proceso inherente e indisociable del desarrollo humano, y como un eje de reflexión permanente sobre los procesos de formación de sus educandos, a partir del estudio crítico de los diferentes supuestos de enseñanza y aprendizaje (...) Hace explícito el principio de la formación integral de sus educandos como la misión y meta final de toda acción pedagógica ${ }^{48}$.

En las dos citas anterior se puede reconocer algunos énfasis muy propios de la pedagogía franciscana, y que se expresan en ideas como: la educación como proceso, la formación integral y la centralidad del estudiante en el proceso educativo. Estos énfasis permiten avizorar

dos grandes referentes, válidos universalmente, para contextualizarlos en su realidad institucional: la filosofía humano-cristiana de la educación y el modelo pedagógico constructivista. El primero porque se ajusta a la vocación cristianan de la Universidad (...) Unido al mensaje del Evangelio, a la espiritualidad franciscana y al legado pedagógico de Madre Beata Caridad Brader (...) De otro lado, la misión de la Universidad también busca formar profesionales "académicamente competentes". Esta teleología institucional obliga a tomar la formación por competencias como un importante referente para guiar los procesos formativos y de aprendizaje (...) Otra sección del modelo describirá la forma como la Universidad Mariana ha adoptado la perspectiva del modelo pedagógico constructivista ${ }^{49}$.

Se habla, explícitamente de poner a dialogar tres enfoques pedagógicos: el primero, emerge del humanismo cristiano y franciscano unido al legado pedagógico de la Beata Caridad Brader Zahaner; el segundo corresponde a un enfoque por competencias y el tercero al enfoque constructivista. Sin embargo, los enfoques pedagógicos de Unimar hace parte de su identidad misma «dado que en su trayectoria educativa ha logrado configurar una

\footnotetext{
${ }^{46}$ Arturo Bolaños Martínez y Carlos Eduardo López Dávila, eds., Escenarios Investigativos (San Juan de Pasto: Editorial Universidad CESMAG, 2020), 15.

${ }^{47}$ Universidad Mariana, Proyecto Educativo Institucional (San Juan de Pasto: Editorial Unimar, 2011), 18-19.

${ }^{48}$ Universidad Mariana, Proyecto Educativo Institucional, 19-20.

${ }^{49}$ Universidad Mariana, Proyecto Educativo Institucional, 20-21.
} 


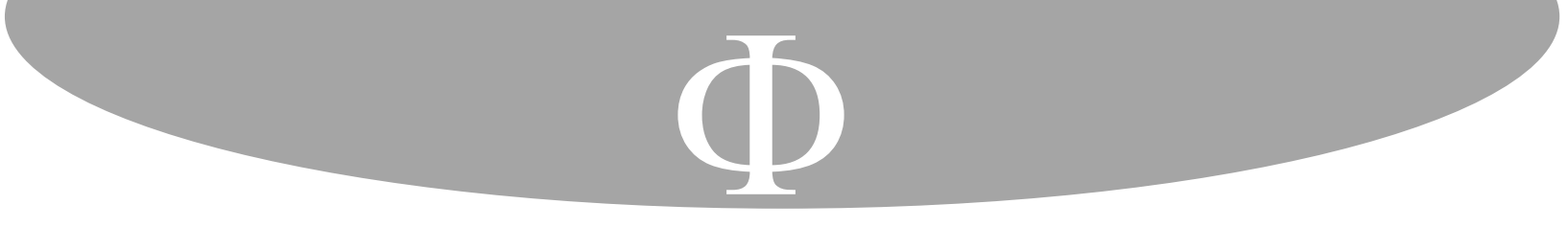

paralelo al reconocimiento y a la identidad que la Universidad logre en el ámbito local y nacional e internacional» ${ }^{61}$.

UNIMAR. Desde la Oficina de Relaciones Nacionales e Internacionales, surge el «Acuerdo 003 del 12 de marzo de 2020» ${ }^{62}$, que actualiza la política de internacionalización presente ya en el PEI (cap. 7) y en el Plan de Desarrollo Institucional 2014-2020 (cap. 5). Dicha Política concibe la internacionalización en una doble dimensión: como cooperación intercultural y como un proceso transversal a las funciones sustantivas; desde allí, define sus líneas estratégicas a saber: internacionalización del currículo, internacionalización de la investigación e internacionalización de la proyección social. De esta manera se acoge a la concepción que sobre internacionalización tiene el MEN, cuando declara que: «la internacionalización es un proceso que confiere una dimensión internacional e intercultural a los mecanismos de enseñanza e investigación de educación superior» ${ }^{63}$.

UNICESMAG. A través de la ORI, entiende la internacionalización como una estrategia para interactuar con otras instituciones de educación superior con el fin de expandir e integrar actividades que involucran a toda la comunidad académica. El PEI, dedica un apartado para presentar los objetivos, las orientaciones y las líneas estratégicas de una "política de internacionalización y de visibilidad regional, nacional e internacional» ${ }^{64}$. Es de resaltar que esta institución, por sus raíces mismas en el padre Guillermo de Castellana, de origen italiano, ha puesto especial interés en esta política, la que concibe como,

lazos de cooperación e integración que beneficia a su comunidad educativa: estudiantes, docentes, administrativos y egresados y como un proceso transversal a las funciones misionales de docencia, investigación y proyección social, con el compromiso de formar personas con visión nacional e internacional y con un sentido crítico, humanístico, multicultural e intercultural para realizar e incidir en su entorno local, nacional y global ${ }^{65}$.

De acuerdo con la cita anterior, la política de internacionalización de Unicesmag muestra las dos caras de un misma moneda: por un lado es una estrategia, y por el otro un proceso de extensión; como estrategia, establece una serie de acciones encaminadas a visibilizar la institución mediante la cooperación con otras instituciones amigas que le ayudan a alcanzar sus fines propios; y como proceso, transversaliza las funciones sustantivas que

\footnotetext{
${ }^{61}$ Universidad Católica Luis Amigó, Proyecto Educativo Institucional. Acuerdo 05 de 2019.

62 Universidad Mariana, Política de Internacionalización. Acuerdo 003 del 12 de marzo de 2020. Por el cual se aprueba la Política de Internacionalización de la Universidad Mariana (San Juan de Pasto: 2020), 1, consultada en septiembre 25, 2020, http://www.umariana.edu.co/docinstitucionales/Acuerdo003-12-032020.pdf.

63 Ministerio de Educación Nacional, Internacionalización de la Educación Superior: Intercambio y Cooperación de Colombia para el mundo, consultada en octubre 20, 2020, https://www.mineducacion.gov.co/1621/article-

217756.html\#: :text=\%C2\%BFQu\%C3\%A9\%20es\%201a\%20internacionalizaci\%C3\%B3n\%20de,mundo\%2 0cada\%20vez\%20m\%C3\%A1s\%20globalizado.

${ }^{64}$ Universidad Centro de Estudios Superiores María Goretti, Proyecto Educativo Institucional.

${ }^{65}$ Universidad Centro de Estudios Superiores María Goretti, Proyecto Educativo Institucional.
} 


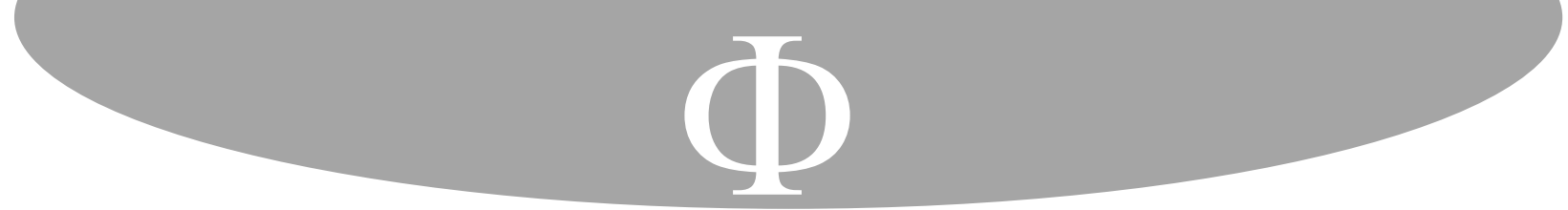

las familias franciscanas, con los múltiples medios que les da la vida en comunidad, habrían de animarse a construir redes de información (...) Hemos de salir de nuestro pequeño ámbito para trabajar en la realidad de un mundo interconectado precisamente para el logro del bien, para la erradicación de la violencia y todas sus dolorosas secuelas. En ese camino está todo prácticamente por hacer y se presenta ante nosotros como un reto de beneficios incalculables (...) $\mathrm{Si}$ «los hijos de las tinieblas» se unen para lograr un mayor beneficio, ¿por qué los que apreciamos a Jesús de Nazaret y a Francisco de Asís no nos vamos a unir con quienes tienen ánimo para enmarcar su actividad económica en tales parámetros? Y, como decimos, no se trata de hacer esto por razón únicamente de la eficacia, sino por la de sentido y coherencia de vida ${ }^{69}$.

\section{Bibliografía}

Aizpurúa Donazar, Fidel. Retos del Franciscanismo para el siglo XXI. Madrid: Escuela Superior de Estudios Franciscanos, 2010.

Bauman, Zygmunt. «El desafío ético de la globalización». Diario El País, sección Opinión, Madrid, (19 de julio de 2001). Consultada en agosto 15 de 2020. https://elpais.com/diario/2001/07/20/opinion/995580007_850215.html

Bolaños Martínez, Arturo y López Dávila, Carlos Eduardo, eds. Escenarios Investigativos. San Juan de Pasto: Editorial Universidad CESMAG, 2020.

Cardona Ramírez, Carlos Mario. La Paideia franciscana en la Universidad de San Buenaventura en Colombia. [Diplomado en Identidad Institucional: Fundamentos del Franciscanismo]. Medellín, Colombia, julio 7-17 de 2020. Consultada diciembre 04 de 2020. https://www.usb.edu.co/index.php/institucional-universidad-sanbuenaventura/documentos-institucionales/estatuto/item/1860-noticias

Cardona Ramírez, Carlos Mario; Muñoz Gaviria, Diego Alejandro; Álvarez Torres, Jair Hernando y Velásquez Moreno, Julián Eduardo. La Paideia Francisana: una mirada a la expansión humana. Medellín: Departamento de Publicaciones USB, 2007.

Castrillón Rincón, Jairo Orlando. «Orientaciones a los modelos educativos desde una perspectiva humanista, y católica». En Orientaciones a los modelos educativos desde la perspectiva humanista, la identidad y los valores católicos: II Encuentro de la Subregión Andina, editado por Stella Valbuena García, 47-58. Santa Cruz de la Sierra: Editorial Universidad Católica de Colombia, 2020.

Conferencia Mundial de Educación Superior. «Las nuevas dinámicas de la Educación Superior y de la investigación para el cambio social y el desarrollo. Comunicado final». Perfiles Educativos Vol. 31 (2009): 119-126. Consultada en marzo de 2020. http://www.scielo.org.mx/pdf/peredu/v31n126/v31n126a8.pdf

Congregación para la Educación Católica. «Educar para el humanismo solidario». Revista de la Universidad de la Salle Vol. 76 (2018): 13-28. Consultada en octubre 16, 2020. https://ciencia.lasalle.edu.co/cgi/viewcontent.cgi?article=2183\&context=ruls

Corbin, J. \& Strauss, A. (2014). Basic of Qualitative Research: Techniques and Procedures for Developing Grounded Theory (4th Editions): SAGE Publications, Inc.

\footnotetext{
${ }^{69}$ Fidel Aizpurúa Donazar, Retos del Franciscanismo para el siglo XXI (Madrid: Escuela Superior de Estudios Franciscanos, 2010), 31 y 34.
} 


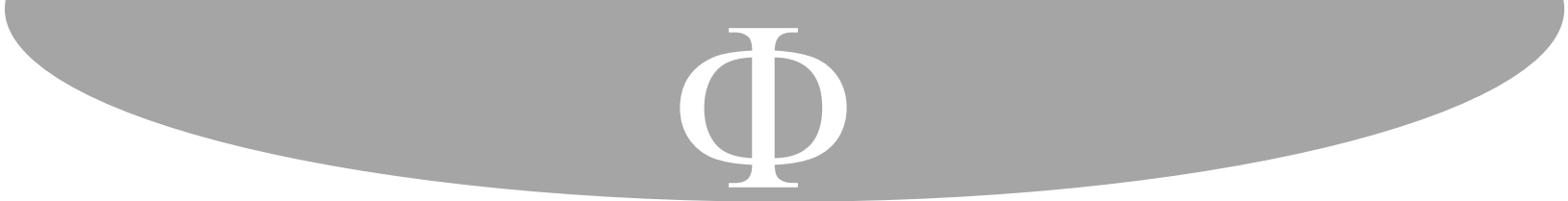

Correa Gómez, Darío. «Algo sobre pedagogía Franciscana». Itinerario Educativo: Revista de la Facultad de Educación Vol. 43-44 (2008): 43-51.

Freyer, Johannes. «Los caminos educativos franciscanos». Carthaginensia: Revista de Estudios e Investigación Vol. 24 (2008): 339-352.

Galeano Atehortúa, Adolfo. La Universidad Franciscana. Evangelización y Posmodernidad. Medellín: Editorial de la Universidad de San Buenaventura, 2004.

González Pérez, Fidenciano. Léxico amigoniano. Diccionario de la pedagogía amigoniana. Madrid: Terciarios Capuchinos Provincia Luis Amigó, 2004.

Iafrancesco, Giovanni Marcello. Nuevos fundamentos para la transformación curricular. A propósito de los estándares. Bogotá: Editorial Delfín, 2003.

Iriarte, Lázaro. Escritos de San Francisco y Santa Clara de Asís. Valencia: Editorial Asís, 1999.

Londoño Orozco, Ernesto. «"De la Ética Mundial” a la "Fraternidad Universal”. La respuesta franciscana al mundo de la globalización y a la heterogeneidad de las culturas». Revista El Ágora USB: Ciencias Sociales Universidad de San Buenaventura. Medellín Vol. 9 (2009): 571-591.

Madariaga, Bernardo. «La pedagogía franciscana y el actual momento pedagógico». Verdad y Vida: Revista de las ciencias del Espíritu Vol. 4 (1946): 177-219.

Martínez Fresneda, Francisco. «El pensamiento Franciscano en la Cultura y en la Universidad». Carthaginensia. Revista de Estudios e Investigación Vol. 14 (1998): 117-142.

Martínez Pérez, Marino. «Nuevos escenarios, perspectivas y desafíos que plantean hoy la misión de la Iglesia y de la Congregación de Religiosos Terciarios Capuchinos». En Congreso Internacional de Pedagogía Amigoniana. Perspectivas y desafíos del siglo XXI, editado por Sandra Juliet Clavijo Zapata, 7-18. Medellín: Fondo Editorial Universidad Luis Amigó, 2018. Consultada en octubre 20 de 2020. https://www.funlam.edu.co/uploads/fondoeditorial/432_Congreso_Internacional_de _Pedagogia_Amigoniana.pdf

Marroquín Yerovi, Mariana; Trejo Chamorro, Héctor; Guerrero Torres, Luis Alfredo y Valverde Riascos, Oscar. Modelo pedagógico. San Juan de Pasto: Editorial Unimar, 2016.

Merino, José Antonio. Humanismo Franciscano: Franciscanismo y Mundo actual. Madrid: Ediciones Cristiandad, 1982.

Ministerio de Educación Nacional. «Internacionalización de la Educación Superior». Consultada en octubre 5 de 2020. Consultada en octubre 20 de 2020. https://www.mineducacion.gov.co/1621/article-196472.html

Ministerio de Educación Nacional. «Internacionalización de la Educación Superior: Intercambio y Cooperación de Colombia para el mundo». Consultada en octubre 20 de 2020. $\quad$ https://www.mineducacion.gov.co/1621/article217756.html\#: :text=\%C2\%BFQu\%C3\%A9\%20es\%201a\%20internacionalizaci\%C 3\%B3n\%20de, mundo\%20cada\%20vez\%20m\%C3\%A1s\%20globalizado.

Ministerio de Educación Nacional. Sistema Nacional de Información de la Educación Superior - SNIES -, «Información poblacional. Estadísticas históricas de la Educación Superior en Colombia». Consultada en noviembre 20 de 2020. https://hecaa.mineducacion.gov.co/consultaspublicas/content/poblacional/index.jsf 


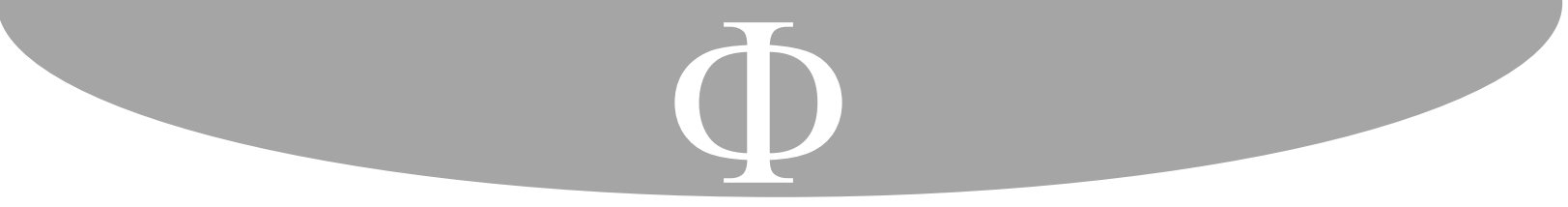

Montealegre González, Alberto, ed. Modelo pedagógico. Referentes conceptuales, lineamientos curriculares y de flexibilidad. Bogotá: Unidad de Publicaciones, Universidad de San Buenaventura, 2010.

Montealegre González, Alberto. «Disertación sobre pedagogía franciscana». Disertación, Universidad de San Buenaventura [inédita], Bogotá, 2010.

Orden de Hermanos Menores. «El Señor nos habla en el Camino. Documento final del Capítulo General Extraordinario de la Orden de Frailes Menores, 2016». Consultada en septiembre 5 de 2020. http://www.franciscanos.org/docoficial/senornoshabla.htm

Orden de Hermanos Menores. Id y enseñad: Directrices Generales para la educación Franciscana. Roma: Curia generale dei Frati Minori, 2009.

Papa Juan Pablo II. «Constitución Apostólica Ex Corde Ecclesiae. Sobre las Universidades Católicas», 1990. Consultada en noviembre 20 de 2020. http://www.vatican.va/content/john-paul-ii/es/apost_constitutions/documents/hf_jpii_apc_15081990_ex-corde-ecclesiae.html

Papa Pablo VI. «Carta Encíclica Populorum Progressio. Sobre la necesidad de promover el desarrollo de los pueblos», 1967. Consultada en noviembre 20 de 2020. http://www.vatican.va/content/paul-vi/es/encyclicals/documents/hf_pvi_enc_26031967_populorum.html

Proyecto Educativo InstitucionaL. San Juan de Pasto: Editorial Unimar, 2011.

Rodríguez Carballo, José. «Los estudios en clave franciscana». Cátedra Guillermo de Ockham de los programas de filosofía y teología de la Universidad de San Buenaventura, Bogotá, 2020. Consultada en noviembre 20 de 2020. https://www.facebook.com/usbdebogota/videos/619321995409393

Soto Forero, Benjamín. «Hacia una pedagogía Franciscana». Revista El Ágora USB: Ciencias Sociales Universidad de San Buenaventura, Medellín Vol. 8 (2008): 459468.

Universidad Católica Luis Amigó. Proyecto Educativo Institucional. Acuerdo Superior N. 05 de 2019. Medellín: Fondo Editorial Universidad Católica Luis Amigó, 2019. $\begin{array}{lllll}\text { Consultada } & \text { en } & \text { septiembre } & 20 & \text { de }\end{array}$ https://www.funlam.edu.co/uploads/documentosjuridicos/684_PEI-ProyectoEducativo-Institucional-2019.pdf

Universidad Centro de Estudios Superiores María Goretti. Proyecto Educativo Institucional. Acuerdo N. 017 de 2020 del Consejo Directivo. San Juan de Pasto: Editorial UNICESMAG, 2020, consultada en marzo 27 de 2021, https://www.unicesmag.edu.co/wp-content/uploads/2021/01/PEI-UNICESMAG2020.pdf

Universidad de San Buenaventura. «Políticas Corporativas. Acreditación Institucional Multicampus». Bogotá, 2016. Consultada en septiembre 25 de 2020. https://www.usb.edu.co/files/renovacion\%20documentos/Pol\%C3\%ADticas\%20Co rporativas\%202016.pdf

Universidad de San Buenaventura. Proyecto Educativo Bonaventuriano. Bogotá: Editorial Bonaventuriana, 2010.

Universidad Mariana. «Política de Internacionalización. Acuerdo 003 del 12 de marzo de 2020. Por el cual se aprueba la Política de Internacionalización de la Universidad 


\section{$\Phi$}

Mariana». San Juan de Pasto, 2020. Consultada en septiembre 25 de 2020. http://www.umariana.edu.co/docinstitucionales/Acuerdo003-12-03-2020.pdf

Vanegas Carvajal, Edgar Alonso. «Identidad de la Universidad Franciscana en Colombia. Aportes desde la pedagogía». I Congreso Internacional de pensamiento ético. Una mirada interdisciplinaria, Universidad CESMAG, San Juan de Pasto, del 16 al 19 de noviembre de 2020.

Zambrano Leal, Armando. De la pedagogía a las ciencias de la educación: Debates y tránsitos. Barcelona: Universitat Oberta de Catalunya, 2019.

Zavalloni, Roberto. Pedagogía franciscana Sviluppi e prospettive. Assisi: Edicione Poziuncola, 1995.

Enviado: 13 de diciembre de 2020

Aceptado: 7 de febrero de 2021 